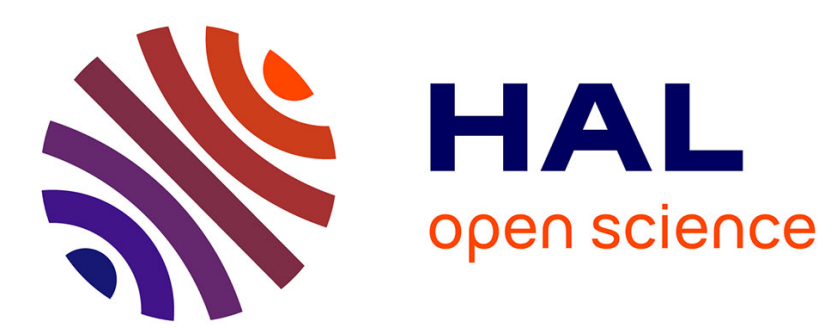

\title{
Is helicity relevant for large scale steady state three-dimensional turbulence?
}

Annick Pouquet, J.-D. Fournier, P.-L. Sulem

\section{To cite this version:}

Annick Pouquet, J.-D. Fournier, P.-L. Sulem. Is helicity relevant for large scale steady state three-dimensional turbulence?. Journal de Physique Lettres, 1978, 39 (13), pp.199-203. 10.1051/jphyslet:019780039013019900 . jpa-00231478

\section{HAL Id: jpa-00231478 https://hal.science/jpa-00231478}

Submitted on 1 Jan 1978

HAL is a multi-disciplinary open access archive for the deposit and dissemination of scientific research documents, whether they are published or not. The documents may come from teaching and research institutions in France or abroad, or from public or private research centers.
L'archive ouverte pluridisciplinaire HAL, est destinée au dépôt et à la diffusion de documents scientifiques de niveau recherche, publiés ou non, émanant des établissements d'enseignement et de recherche français ou étrangers, des laboratoires publics ou privés. 


\title{
LE JOURNAL DE PHYSIQUE-LETTRES
}

Classification

Physics Abstracts

$47.25 \mathrm{C}$

\section{IS HELICITY RELEVANT FOR LARGE SCALE STEADY STATE THREE-DIMENSIONAL TURBULENCE ?}

\author{
A. POUQUET, J.-D. FOURNIER and P.-L. SULEM
}

Observatoire de Nice, B.P. no 252, 06007 Nice Cedex, France

(Reçu le 28 mars 1978, accepté le 18 mai 1978)

\begin{abstract}
Résumé. - On présente un calcul de groupe de renormalisation pour une turbulence forcée hélicitaire. On montre que : (i) l'hélicité produit un terme supplémentaire, proportionnel à rot $\nabla^{2} v$ $(v=$ vitesse) qui est une perturbation non pertinente de l'amortissement visqueux; (ii) la partie hélicitaire de la force n'est jamais plus qu'une perturbation marginale; (iii) quand on considère les équations de la magnétohydrodynamique au lieu des équations de Navier-Stokes, l'hélicité génère une perturbation pertinente de l'amortissement Joule, proportionnelle à rot $b$ ( $b=$ champ magnétique.
\end{abstract}

\begin{abstract}
A renormalization group calculation of forced helical turbulence is presented. It is shown that : (i) helicity produces an additional term, proportional to curl $\nabla^{2} v(v=$ velocity), which is an irrelevant perturbation of the viscous damping; (ii) the helical part of the forcing is never more than a marginal perturbation; (iii) when the magnetohydrodynamics equations are used instead of the Navier-Stokes equations, helicity generates a relevant perturbation of the Joule damping, proportional to $\operatorname{curl} b$ ( $b=$ magnetic field).
\end{abstract}

1. Introduction. - The renormalization group (RG) technique in its dynamical version [1] has recently been applied to turbulence by Forster, Nelson and Stephen [2]. They studied the large scale, low frequency properties of a homogeneous, isotropic, incompressible turbulence stirred by random forces. Here, we consider the case of (three-dimensional) helical turbulence, i.e. a turbulence which is invariant under translations and rotations but not under plane reflections. This implies the non-vanishing of the helicity, defined as the correlation between the velocity $v$ and the vorticity curl $v$. Helicity is an invariant of the inviscid three-dimensional NavierStokes (NS) equations [3].

The NS equations read

$$
\begin{gathered}
\frac{\partial v}{\partial t}(\mathbf{x}, t)+\lambda_{0}[(v \cdot \nabla) v+\nabla p]=v_{0} \nabla^{2} v+f(\mathbf{x}, t) \\
\nabla \cdot v=0
\end{gathered}
$$

where $p$ denotes the pressure, $v_{0}$ the viscosity and $\lambda_{0}$ a formal expansion parameter which measures the strength of the non-linear terms. The force $f$ is assumed to be a zero-mean Gaussian random function, whose Fourier transform has a correlation given by

$$
\begin{aligned}
& \left\langle\hat{f}_{i}(\mathbf{k}, \omega) \hat{f}_{j}\left(\mathbf{k}^{\prime}, \omega^{\prime}\right)\right\rangle= \\
& \quad=2 D_{0}(2 \pi)^{4} \mathcal{F}(\mathbf{k}) P_{i j}(\mathbf{k}) \delta\left(\mathbf{k}+\mathbf{k}^{\prime}\right) \delta\left(\omega+\omega^{\prime}\right) \\
& +2 \tilde{D}_{0}(2 \pi)^{4} \tilde{\mathcal{F}}(k) \varepsilon_{i j l} k_{l} \delta\left(\mathbf{k}+\mathbf{k}^{\prime}\right) \delta\left(\omega+\omega^{\prime}\right) .
\end{aligned}
$$

$P_{i j}(\mathbf{k})=\delta_{i j}-k_{i} k_{j} / k^{2}$ is the incompressibility projection operator and $\varepsilon_{i j l}$ the fundamental antisymmetric tensor. $D_{0}$ and $\tilde{D}_{0}$ are positive and measure the intensity of the energy and helicity injection. A Schwarz inequality implies that

$$
\frac{\tilde{D}_{0} k|\tilde{\mathfrak{F}}(k)|}{D_{0} \widetilde{F}(k)} \leqslant 1
$$

the equality corresponding to a maximal helicity injection. We also introduce an ultraviolet wavenumber cut-off $\Lambda$ (corresponding, say, to the viscous cut-off), beyond which velocity and forcing are assumed to vanish.

It appears impossible to analytically continue the helicity to space dimensions $d$ other than three, because of the curl operator involved in its definition. We cannot therefore use as in Forster et al. [2] an expansion in $\varepsilon=4-d$ and it is necessary to exhibit a crossover which is not related to the space-dimen- 
sionality. This has been done by Fournier [4] for non-helical turbulence. Let us indicate how it can be set up. With a suitable change of variables, one can regroup the different parameters which enter equations (1) and (2) (with $\tilde{D}_{0}=0$ ) into a single reduced interaction parameter.

$$
\bar{\lambda}_{0}=\lambda_{0} D_{0}^{1 / 2} v_{0}^{-3 / 2} .
$$

Assuming a modal injection spectrum $\mathcal{F}(k)=k^{-r^{\prime}}$ for small $k$, dimensional analysis yields

$$
\left[\bar{\lambda}_{0}\right]=[\text { length }]^{\beta} \text { with } \beta=\frac{d-4-r^{\prime}}{2} .
$$

The dimensionless parameter playing the role of a local Reynolds number is then $\bar{\lambda}_{0}(k)=\bar{\lambda}_{0} k^{\beta}$. The infrared behaviour of this local interaction parameter is governed by the sign of the crossover parameter $\beta$. For $\beta>0, \bar{\lambda}_{0}(k)$ tends to zero as $k \rightarrow 0$, and equation (1) reduces to a linear Langevin equation, while for $\beta<0$ the non-linear terms become increasingly important as $k \rightarrow 0$. For $\beta$ slightly negative, one is faced with a singular perturbation problem which the RG is designed to handle [4]. In the case of isotropic turbulence, one can get rid of the space dimension in $\beta$ by introducing the injection spectrum per unit wavenumber

$$
F(k)=S_{d} k^{d-1} \mathcal{F}(k),
$$

obtained by integration of the injection spectrum per wavevector $\mathcal{F}(k)$ over a sphere of diameter $k$. In equation (5), $S_{d}$ is the surface of the $d$-dimensional unit sphere. The power law dependence of $F(k)$ as $k \rightarrow 0$ is given by $F(k) \propto k^{-r}$ with $r=r^{\prime}-d+1$. The crossover parameter becomes

$$
\beta=-(3+r),
$$

which is independent of the dimension. This allows one to work at a fixed space dimension and to take $\varepsilon=r+3$ as the expansion parameter.

In the helical case, we are thus led to introduce the helicity injection spectrum per wavenumber [5]

$$
\tilde{F}(k)=4 \pi k^{4} \tilde{\mathcal{F}}(k) \propto k^{-\tilde{r}}
$$

together with the energy injection per wavenumber

$$
F(k)=4 \pi k^{2} \mathcal{F}(k) \propto k^{-r},
$$

with $\tilde{r} \leqslant r-1$. The helicity injection will be said to be relatively maximal, if $\tilde{D_{0}} \widetilde{F}(k) / D_{0} k F(k)$ remains of order unity for $k \rightarrow 0$, i.e. if $\tilde{r}=r-1$, with $\widetilde{D}_{0} \leqslant D_{0}$.

\section{Renormalization group procedure for helical tur-} bulence. - The three-dimensional NS equations (1) written for the Fourier components of the velocity field with wavenumber $0 \leqslant k \leqslant \Lambda$ read :

$$
\begin{aligned}
\hat{v}_{l}(\mathbf{k}, \omega)=G_{0}(k, \omega) & \hat{f}_{l}(\mathbf{k}, \omega)-\frac{i}{2} \lambda_{0} G_{0}(k, \omega) P_{l m n}(\mathbf{k}) \times \\
& \times \int_{q \Omega} \hat{v}_{m}(\mathbf{q}, \Omega) \hat{v}_{n}(\mathbf{p}, \omega-\Omega) .
\end{aligned}
$$

In equation (9),

$$
G_{0}(k, \omega)=\left(-i \omega+v_{0} k^{2}\right)^{-1}
$$

denotes the free propagator and

$$
P_{l m n}(\mathbf{k})=k_{n} P_{l m}(\mathbf{k})+k_{m} P_{l n}(\mathbf{k}) .
$$

Furthermore, the following notation has been used :

$$
\int_{q \Omega}=\int_{\substack{|\mathbf{q}|<\Lambda \\ \mathbf{p}+\mathbf{q}=\mathbf{k} ;|\mathbf{p}|<\Lambda}} \frac{\mathrm{d}^{3} q}{(2 \pi)^{3}} \int_{-x}^{+x} \frac{\mathrm{d} \Omega}{2 \pi} .
$$

The dynamical $R G$ procedure is discussed in detail in [1] and [2]. An introduction in a slightly different spirit (singular perturbation) is given in [4]. The first step consists in the elimination of the modes $v^{>}(\mathbf{k}, \omega)$ such that $\Lambda \mathrm{e}^{-l} \leqslant k \leqslant \Lambda$. This is done by solving equation (9) for $v^{>}$as a power series in $\lambda_{0}$ and substituting it into the equation for $v^{<}(k, \omega)$ for which $k<\Lambda \mathrm{e}^{-l}$. This is conveniently done by using the following diagramatic representation

$$
\begin{aligned}
& \frac{k,(1)}{\left.\mathbf{k} \cdot()^{\prime \prime}\right)(\omega)^{\prime \prime}}=G_{0}(k, \omega) \\
& { }_{i}^{\prime \prime} \mathrm{O}_{\bar{i}}^{\mathbf{h} \cdot()^{\prime}}=(2 \pi)^{4} D_{0} \mathcal{F}(k) P_{i j}(\mathbf{k}) \delta\left(\mathbf{k}+\mathbf{k}^{\prime}\right) \delta\left(\omega+\omega^{\prime}\right) \\
& { }_{i}^{\mathbf{k} . \prime \prime} \Theta_{i}^{\mathbf{k}^{\prime} \cdot\left(\omega^{\prime}\right.}=(2 \pi)^{4} \tilde{D}_{0} \tilde{\mathcal{F}}(k) \varepsilon_{i j l} k_{l} \delta\left(\mathbf{k}+\mathbf{k}^{\prime}\right) \delta\left(\omega+\omega^{\prime}\right) \\
& \stackrel{\omega, \mathbf{k}}{\rightarrow} \underbrace{m \mathbf{q} . \Omega}_{l p, \omega}=-\frac{\lambda_{0}}{2} i P_{l m n}(\mathbf{k}) \int_{q, \Omega} \\
& \stackrel{\mathbf{k}, \omega}{\underset{i}{\longrightarrow}}=\hat{v}_{j}(\mathbf{k}, \omega) \text {. }
\end{aligned}
$$

The elimination procedure generates many terms in the equation for $v^{<}$, but at the lowest non trivial order, namely $0\left(\lambda_{0}^{2}\right)$, the terms which are relevant in the limit $k \rightarrow 0$ lead to

(i) A renormalization of the viscous term $v k^{2} \hat{v}(\mathbf{k}, \omega)$ which becomes

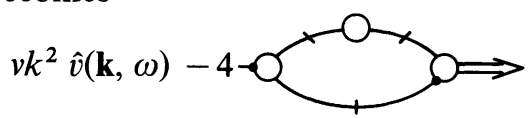

The dashed line + indicates that the corresponding wavenumber belongs to the shell $\Lambda \mathrm{e}^{-l} \leqslant q \leqslant \Lambda$ and is consequently integrated over. One checks that the diagram appearing in (13) is proportional to $k^{2} \hat{v}(\mathbf{k}, \omega)$ for $k \rightarrow 0$.

Remark. - Helicity generates an extra linear term,

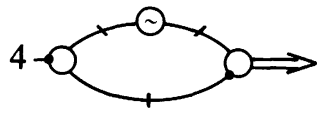

which is proportional to $k^{2} \mathbf{k} x \hat{v}(\mathbf{k}, \omega)$, or equivalent- 
ly, in configuration space, to curl $\nabla^{2} v(\mathbf{x}, t)$. This term is irrelevant in the limit $k \rightarrow 0$ (see also section 3 ). (ii) A renormalization of the random forces whose correlation function $\left\langle\hat{f}_{i}(k, \omega) \hat{f}_{j}\left(k^{\prime}, \omega^{\prime}\right)\right\rangle$ becomes

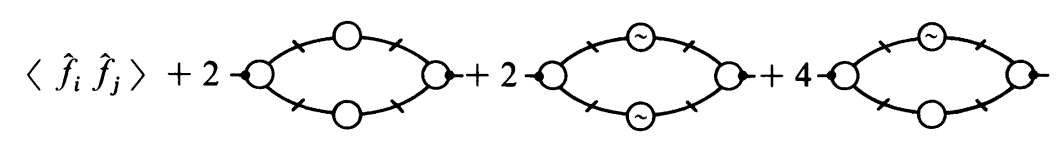

The first two diagrams are proportional to $k^{2} P_{i j}(\mathbf{k})$ and contribute to the renormalization of the nonhelical part of the force correlation when $r \leqslant-4$ (Eq. (8)), while the third diagram is proportional to $k^{2} \varepsilon_{i j l} k_{l}$ and contributes to the renormalization of its helical part when $\tilde{r} \leqslant-6$ (Eq. (7)).

In the non-helical case, the sum of the terms which renormalize the vertex is of higher order in $k$ than the bare vertex and is therefore irrelevant. The extra terms generated by helicity are individually irrelevant.

The computation of the above diagrams leads after some algebra to the intermediate recursion relations written for $r \geqslant-4$ and $\tilde{r} \geqslant-6$ (with the constraint $\tilde{r} \leqslant r-1)$. The case $\tilde{r}<-4$ or/and $\tilde{r}<-6$ will be discussed later.

$$
\begin{aligned}
& \lambda_{\mathrm{I}}(l)=\lambda_{0} \\
& D_{\mathrm{I}}(l)=D_{0}\left[1-\delta_{r,-4} \frac{\bar{\lambda}_{0}^{2}}{12 \pi^{2}}\left\{\frac{7}{5} \frac{1-\mathrm{e}^{(2 r+7) l}}{2 r+7}-\frac{\tilde{D}_{0}}{D_{0}} \times \frac{1-\mathrm{e}^{(2 \tilde{r}+9) l}}{2 \tilde{r}+9}\right\}\right] \\
& \tilde{D}_{\mathrm{I}}(l)=\tilde{D}_{0}\left[1-\delta_{\tilde{r}_{,}-6} \bar{\lambda}_{0}^{2} \frac{r+6}{90 \pi^{2}} \frac{1-\mathrm{e}^{l(r+\tilde{r}+9)}}{r+\tilde{r}+9}\right] \\
& v_{\mathrm{I}}(l)=v_{0}\left[1-\bar{\lambda}_{0}^{2} \frac{3-r}{60 \pi^{2}} \frac{1-\mathrm{e}^{(r+3) l}}{r+3}\right] .
\end{aligned}
$$

In equation (16) $\delta_{\alpha, \beta}$ denotes the Kronecker symbol.

The second step of the RG procedure consists in rescaling the wavenumbers, frequencies and remaining velocity components :

$$
\mathbf{k}^{\prime}=\mathrm{e}^{l} \mathbf{k}, \quad \omega^{\prime}=\exp \left[\int_{0}^{l} z\left(l^{\prime}\right) \mathrm{d} l^{\prime}\right] \omega, \quad v^{<}(\mathbf{k}, \omega)=\exp \left[\int_{0}^{l} \tau\left(l^{\prime}\right) \mathrm{d} l^{\prime}\right] v^{\prime}\left(\mathbf{k}^{\prime}, \omega^{\prime}\right) .
$$

This induces the following scaling on the parameters which enter the equation for $v^{<}$:

$$
\begin{aligned}
& \lambda^{\prime}(l)=\lambda_{\mathrm{I}}(l) \exp \int_{0}^{l}\left[\tau\left(l^{\prime}\right)-4\right] \mathrm{d} l^{\prime} \\
& D^{\prime}(l)=D_{\mathrm{I}}(l) \exp \int_{0}^{l}\left[3 z\left(l^{\prime}\right)+(r+5)-2 \tau\left(l^{\prime}\right)\right] \mathrm{d} l^{\prime} \\
& \tilde{D}^{\prime}(l)=\tilde{D}_{\mathrm{I}}(l) \exp \int_{0}^{l}\left[3 z\left(l^{\prime}\right)+(\tilde{r}+6)-2 \tau\left(l^{\prime}\right)\right] \mathrm{d} l^{\prime} \\
& v^{\prime}(l)=v_{\mathrm{I}}(l) \exp \int_{0}^{l}\left[z\left(l^{\prime}\right)-2\right] \mathrm{d} l^{\prime} .
\end{aligned}
$$

Combining (15)-(17) and replacing $l$ by an infinitesimal parameter [2], we finally obtain the differential recursion relations 


$$
\begin{aligned}
\frac{\mathrm{d} \lambda(l)}{\mathrm{d} l} & =\lambda(l)[\tau(l)-4] \\
\frac{\mathrm{d} D(l)}{\mathrm{d} l} & =D(l)\left[3 z(l)+r+5-2 \tau(l)+\delta_{r,-4} \frac{\bar{i}^{2}(l)}{12 \pi^{2}}\left(\frac{7}{5}-\frac{\tilde{D}(l)}{D(l)}\right)\right] \\
\frac{\mathrm{d} \tilde{D}}{\mathrm{~d} l}(l) & =\tilde{D}(l)\left[3 z(l)+\tilde{r}+6-2 \tau(l)+\delta_{\tilde{r},-6} \frac{r+6}{90 \pi^{2}} \bar{\lambda}^{2}(l)\right] \\
\frac{\mathrm{d} v}{\mathrm{~d} l}(l) & =v(l)\left[z(l)-2+\frac{3-r}{60 \pi^{2}} \bar{\lambda}^{2}(l)\right]
\end{aligned}
$$

from which we derive that the reduced interaction parameter

$$
\bar{\lambda}(l)=\lambda(l) D^{1 / 2}(l) v^{-3 / 2}(l)
$$

which satisfies :

$$
\begin{aligned}
& \frac{\mathrm{d} \bar{\lambda}}{\mathrm{d} l}(l)=\bar{\lambda}(l) \times \\
& \times\left[\frac{\varepsilon}{2}-\frac{3-r}{40 \pi^{2}} \bar{\lambda}^{2}+\delta_{r,-4} \frac{\bar{\lambda}^{2}}{12 \pi^{2}}\left(\frac{7}{5}-\frac{\tilde{D}(l)}{D(l)}\right)\right],
\end{aligned}
$$

with $\varepsilon=r+3$.

We have then to determine the asymptotic behaviour of the scaling factors in order that the solution of the system (18)-(20) tends to a fixed point. Different cases must be distinguished according to the values of $r$ and $\tilde{r}$ but the conclusion is always the same. Helicity does not destabilize the non-helical fixed point for $\lambda, v$ and $D$, and consequently does not change the scaling factors. It follows that the large scale behaviour of the energy spectrum

$$
\begin{aligned}
E(k) \propto \int \mathrm{d} \mathbf{k}^{\prime} \mathrm{d} \omega^{\prime} \mathrm{d} \omega k^{2} \times \\
\quad \times \sum_{i=1}^{3}\left\langle\hat{v}_{i}(\mathbf{k}, \omega) \hat{v}_{i}\left(-\mathbf{k}^{\prime},-\omega^{\prime}\right)\right\rangle
\end{aligned}
$$

is the same as in the absence of helicity , namely $[2,4]$ :

$$
\begin{aligned}
r & =-4 & & E(k) \sim k^{2} \\
-4<r & <-3 & & E(k) \sim k^{1-(r+3)} \\
r & =-3 & & E(k) \sim k(\ln 1 / k)^{-1 / 3} \\
r & >-3 & & E(k) \sim k^{1-\frac{2}{3}(r+3)+0\left(\varepsilon^{2}\right)} .
\end{aligned}
$$

Let us now turn to the helicity spectrum

$$
\begin{aligned}
H(k) \propto \int & \mathrm{d} \mathbf{k}^{\prime} \mathrm{d} \omega \mathrm{d} \omega^{\prime} k^{4} \times \\
& \times \sum_{i}\left\langle\hat{v}_{i}(\mathbf{k}, \omega) \varepsilon_{i j l} k_{j} \hat{v}_{l}\left(-\mathbf{k}^{\prime},-\omega^{\prime}\right)\right\rangle .
\end{aligned}
$$

The spectrum will again be said to be relatively maximal if $H(k) / k E(k)$ remains of order unity for $k \rightarrow 0$. It can be checked that when the helicity injection is not relatively maximal (i.e. when $\tilde{r}<r-1$ ),
$\tilde{D}(l) \underset{l \rightarrow 0}{\longrightarrow} 0$, so that the helicity injection becomes an irrelevant perturbation. In contrast, when the helicity injection is relatively maximal (i.e. when $\tilde{r}=r-1$ ), $\tilde{D}(l)$ has a non zero limit but, as already said, this does not affect the energy spectrum : a relatively maximal helicity injection is thus a marginal perturbation. In the latter case, the helicity spectrum is itself relatively maximal.

Finally, let us consider the case of energy and/or helicity injection spectra (per wavevector) steeper than $k^{2}(r<-4$ and/or $\tilde{r}<-6$, with the condition $\tilde{r} \leqslant r-1)$. After elimination of the first high wavenumber shell $\Lambda \mathrm{e}^{-l}<k<\Lambda$, the non helical and/or the helical part of the forcing are negligible in comparison with the renormalized one. Thus, at the second iteration, namely the elimination of the shell $\Lambda \mathrm{e}^{-2 l}<k<\mathrm{e}^{-l} \Lambda$, the recursion formulas will be the same as in the case $r=-4$ and/or $\tilde{r}=-6$. As a consequence, helicity is in this case an irrelevant perturbation even when it is injected at a maximal rate. If injection of energy and/or helicity is confined to a narrow band of wavenumbers, the same conclusions hold.

3. Conclusion. - We have shown in this paper that helicity is at best a marginal perturbation in the Navier-Stokes equations. We also found that, by integrating out the small scales, we generate in the equation for the large-scale velocity field a new linear term proportional to curl $\nabla^{2} v$. This term is negligible in the limit $k \rightarrow 0$ when compared to the (renormalized) dissipation (proportional to $\nabla^{2} v$ ). Nevertheless, for finite wavenumbers, this new term acts as a negative damping which opposes the eddy-viscosity. This suggests a decrease of the eddy-viscosity and hence, an inhibition of energy transfer to the small scales in the presence of helicity. Such an inhibition is in agreement with the analysis of Kraichnan [6], based on the interaction between two helicity waves (see also [7]), and with a two-point closure calculation of André and Lesieur [8].

We finally wish to mention that our conclusions concerning the relevance of helicity seem completely changed if, instead of the NS equations (1), we consider the MHD equations for a conducting fluid 


$$
\begin{gathered}
\frac{\partial v}{\partial t}+(v \cdot \nabla) v=-\nabla p+v \nabla^{2} v+(b \cdot \nabla) b+f^{\mathrm{v}} \\
\frac{\partial b}{\partial t}+(v \cdot \nabla) b=(b . \nabla) v+\eta \nabla^{2} b+f^{\mathrm{M}} \\
\nabla \cdot v=0 ; \quad \nabla \cdot b=0 .
\end{gathered}
$$

The study of large scale non helical MHD turbulence by the renormalization procedure is under way and yields new fixed points (to be published elsewhere). Let us mention the following result concerning MHD helical turbulence. Eliminating the small scale kinetic and magnetic modes, it is easily shown that the renormalized equation for the magnetic field $b$ contains two new linear terms, stemming from diagrams similar to (14) but with Ohm's law vertices and with respectively kinetic and magnetic helical forcing. Both these terms are proportional to $\operatorname{curl} b$ and thus contain a single space derivative, as opposed to the purely hydrodynamical case. As $k \rightarrow 0$, they dominate the renormalized Joule dissipation and are easily checked to have a destabilizing influence on large scale magnetic fields. In MHD turbulence, helicity thus generates a relevant perturbation of the Joule damping. Note that the RG permits one to recover by a systematic procedure the helicity effects (also called " $\alpha$ effects ») previously obtained by more or less ad hoc techniques [5, 9-12]. Closure calculations also indicate that no steady state exists for the large scale because of the presence of an inverse cascade of magnetic helicity $\langle a . b\rangle(a$, magnetic vector potential) [11]. Such an inverse cascade does not appear at this point to be tractable by the $\mathrm{RG}$ procedure.

Acknowledgments. - We are indebted to U. Frisch, M. Lesieur and H. K. Moffatt for useful discussions.

\section{References}

[1] Ma, S. and Mazenko, G. F., Phys. Rev. B 11 (1975) 4077.

[2] Forster, D., Nelson, D. R. and Stephen, M. J., Phys. Rev. Lett. 36 (1976) 867; Phys. Rev. A 16 (1977) 732.

[3] Brissaud, A., Frisch, U., Léorat, J., Lesieur, M. and Mazure, A., Phys. Fluids 16 (1973) 1366.

[4] Fournier, J. D., Thèse de troisième cycle. Université de Nice (1977).

[5] Moffatt, H. K., (1978) Magnetic Field Generation in Electrically Conducting Fluids. Cambridge Monographs on Mechanics and Applied Mathematics (Cambridge University Press).
[6] Kraichnan, R. H., J. Fluid Mech. 59 (1973) 745.

[7] Kraichnan, R. H., J. Fluid Mech. 77 (1976) 753.

[8] André, J. C. and Lesieur, M., J. Fluid Mech. 81 (1977) 187.

[9] Steendeck, M., Krause, F. and Radler, K. H., Z. Naturforsch. 21a (1966) 364

[10] Moffatt, H. K., J. Fluid Mech. 41 (1970) 435.

[11] Pouquet, A., Frisch, U. and LÉorat; J., J. Fluid Mech. 77 (1976) 321.

[12] K NoBloch, E., J. Fluid Mech. 83 (1977) 129. 